\title{
Herança Maldita e Política Monetária em um Modelo de Assimetria de Informação*
}

\author{
Carlos E. S. Gonçalves**
}

Sumário: 1. Introdução; 2. Modelo; 3. Discussão; 4. Conclusão.

Palavras-chave: sinalização; dívida; inflação.

Códigos JEL: E61; E65.

Neste artigo mostramos - usando um modelo de dois períodos com assimetria de informação quanto às preferências do governo - que é possível que uma política monetária restritiva seja capaz de arrefecer as expectativas de inflação ainda que a situação fiscal do país não seja muito sólida. Se por um lado, uma dívida mais elevada pressiona a inflação para cima (efeito de dominância fiscal), por outro ela possibilita que um governo conservador se separe, sob os olhos do público, de um governo gastador. Por conta deste incentivo a sinalizar, inflação esperada e realizada não são positivamente correlacionadas com o estoque de dívida herdada. Assim, a "herança maldita" do governo FHC pode ter ajudado o governo Lula em sua tarefa de sinalizar seu tipo antiinflacionário para a sociedade.

In this paper we use a simple two-period model in which government's preferences are private information to show that a tough monetary stance may help in bringing inflation expectations to lower levels even if the fiscal side of the economy is not in good shape. True, a restrictive monetary policy leads to higher debt levels and potentially to higher expected inflation (fiscal dominance effect). But, on the other hand, it also allows a tough-type government to separate itself from a lax-type one in the eyes of the public (signaling effect). A feature of the model is that inflation and inherited debt are not necessarily positive correlated for all levels of debt. Concretely, the high debt levels Lula's administration inherited from FHC's term in office (the so-called "damned heritage") may have helped the former to signal to markets its degree of inflation aversion.

\footnotetext{
*Artigo recebido em mai. 2004 e aprovado em mai. 2005. Agradeço a Bernardo Guimaraes, Fábio Kanczuk e o parecerista anônimo por seus comentários.

** Universidade de Sao Paulo, Departamento de Economia. E-mail: cesg@usp.br
} 


\section{Introdução}

Em trabalho hoje amplamento reconhecido tanto no meio acadêmico, como entre os gerenciadores de política econômica, Sargent e Wallace (1981) chamaram a atenção para o fato de a eficácia da política monetária ser fortemente dependente, na sua tarefa de manter a estabilidade de preços e coordenar as expectativas de inflação, da robustez do lado fiscal da economia. A lógica, apelidada pela profissão de "aritmética desagradável" ou "dominância fiscal", não é difícil de ser entendida: quando a dívida pública é elevada, um aumento do juro real com vistas a combater um recrudescimento da inflação tem o desgradável efeito colateral de pressionar o endividamento público. Segue-se que, caso os agentes privados passem a julgar uma futura monetização da dívida como inevitável, ${ }^{1}$ as expectativas de inflação se elevarão e o resultado final sobre a inflação corrente poderá ser o contrário do inicialmente almejado. De modo simétrico, endividamento moderado e políticas fiscais prudentes ensejam expectativas de inflação (tudo o mais constante) mais baixas, facilitando, portanto, a tarefa da autoridade monetária em manter a estabilidade de preços.

Baseando-se nestas idéias e na literatura dos modelos de sinalização aplicados à política monetária, ${ }^{2}$ Drazen e Masson (1994) aprimoram e qualificam a lógica da aritmética desagradável ao mostrar, em um modelo com assimetria de informação quanto ao "tipo" (grau de aversão inflacionária) do governo, que um arrocho monetário levará a maior inflação esperada apenas quando o efeito associado ao esforço em sinalizar preferências antiinflacionárias for superado pelo decorrente da aritmética desagradável. ${ }^{3}$

Seguindo a mesma linha de Drazen e Masson, propomos, neste artigo, um modelo que incorpore os trade-offs com que se defronta um novo governo que, ao mesmo tempo que herda de seu predecessor um elevado estoque de dívida pública ("herança maldita"), almeja ser visto pelo setor privado como um tipo "durão" 4 em relação à inflação. A motivação é fornecer um contra-ponto ao recente trabalho de Blanchard (2004), no qual o autor sugere que o agora tão alardeado sistema de metas de inflação é ineficaz na tarefa de estabilizar em baixos patamares as

\footnotetext{
${ }^{1}$ Não necessariamente no sentido de que não haverá recursos suficientes para honrar uma dívida mais elevada, mas, principamente, no sentido de que o governo pode não se mostrar politicamente disposto a elevar impostos ou cortar gastos com vistas a honrar suas obrigações financeiras ("unwillingness to pay").

${ }^{2}$ Backus e Driffil (1985) e Vickers (1986).

${ }^{3}$ No caso do referido artigo, a variável de estado que piora com o aperto monetário não é a dívida pública, mas o desemprego futuro (devido a persistência intertemporal do mesmo).

${ }^{4}$ No sentido proposto por Rogoff (1985).
} 
expectativas de inflação no caso de uma economia altamente endividada como a brasileira. Nosso argumento é que, por mais intuitiva que seja a história de dominância fiscal, ela é no mínimo incompleta para explicar a experiência brasileira do ano de 2003. Primeiro porque a hipótese de que o setor privado buscava, na transição de governo, inferir qual o grau de aversão à inflação do PT observando suas primeiras medidas no campo monetário soa intuitivamente irrefutável ${ }^{5}$ (o que nos leva a conjeturar um papel fundamental para o efeito sinalização). Segundo, porque é difícil conciliar o fato de que as expectativas de inflação se contrairam fortemente já no primeiro trimestre de 2003, com uma história onde os efeitos de aritmética desagradável são os preponderantes.

Com o intuito de aprofundar o debate sobre os trade-offs enfrentados pela autoridade monetária brasileira no ano de 2003, apresentamos aqui um modelo simples de 2 períodos onde há assimetria de informação quanto ao tipo do novo governo. ${ }^{6}$ Mostramos que caso a dívida se situe em um certo intervalo intermediário (endogenamente determinado), um governo do tipo "durão" optará otimamente por implementar uma política monetária mais restritiva que a sua preferida na tentativa de se "separar" de um tipo pouco avesso à inflação (ou "fraco").

Interessantemente, quando a dívida herdada é suficientemente baixa, torna-se infactível para um "durão" convencer o público de suas credenciais anti-inflacionárias. Isto porque, com um baixo endividamento, é pouco custoso também para um governo "fraco" mimetizar a política mais restritiva praticada por aquele. Note-se que neste caso de baixo endividamento, as expectativas de inflação serão mais altas devido à impossibilidade de o público inferir as preferências do recém empossado governante (equilíbrio agregador, ou de pooling).

Também decorre do modelo que a inflação realizada será em média mais baixa no caso de assimetria de informação (relativamente a um benchmark de informação perfeita). Isto porque, para um nível intermediário de endividamento, o governo conservador, no desejo de colher os benefícios de ser visto como tal, praticará uma política monetária extremamente apertada. Isto implicará em menor viés inflacionário e, portanto, maior bem-estar. Em outras palavras, a sinalização acaba funcionando como um "commitment device" quando há discricionariedade na condução da política monetária em um ambiente de informação imperfeita. Já para patamares muito elevados da dívida pública, uma política monetária extremamente restritiva passa a ser muito custosa e, portanto, ambos os "tipos" abandonam qualquer tipo de ação estratégica - o durão de ser extremamente con-

\footnotetext{
${ }^{5}$ Dada a contradição entre as posições históricas do partido e sua plataforma eleitoral.

${ }^{6}$ Um modelo similar ao nosso, porém mais próximo ao sugerido por Drazen e Masson, pode ser encontrado no recente artigo de Benigno e Missale (2004).
} 
servador, e o fraco de mimetizar a política daquele - e implementam sua política monetária preferida.

Finalmente, um outro resultado do modelo é que não só a inflação esperada pode apresentar correlação negativa com o nível de endividamento, mas também a realizada. Isto ocorrerá quando a dívida cruzar o limiar inferior do intervalo intermediário acima mencionado.

\section{Modelo}

Nesta seção desenvolvemos um modelo básico de dois períodos onde o governo, que extrai ganhos em aumentar o gasto público, mas perde utilidade com inflações positivas ou negativas, maximiza seu bem-estar escolhendo de maneira ótima a taxa de inflação em cada período.

Primeiramente, resolvemos o problema do governo em um mundo sem assimetrias informacionais. Contra este benchmark, comparamos os resultados obtidos quando assumimos que o "tipo" do governo é informação privada. Vale dizer que, mesmo em um ambiente de assimetria informacional, ambos os tipos implementarão sua política preferida ( one-shot Nash) no último período, ficando qualquer ação estratégica que vise manipular as expectativas de inflação restrita ao primeiro.

No início do segundo período, a dívida herdada do governo anterior precisa ser honrada, não havendo possibilidade de default explícito. No entanto, o governo é capaz de afetar a taxa de juro real que remunera seus títulos vincendos caso consiga engendrar uma inflação não esperada pelos agentes privados (e, portanto, não imbutida no preço destes papéis). Finalmente, assumimos a hipótese de que não há uma tecnologia de commitment disponível, e também que pelo fato da dívida vencer no início do segundo período, a taxa real que a remunera é função da política monetária decidida no final do primeiro.

\subsection{Preferências}

As preferência do governo são dadas por:

$$
U_{i}=\theta_{i} g_{t}-\frac{m_{t}^{2}}{2}
$$

onde:

$i=T ; W, t=1 ; 2, m$ é inflação, $\mathrm{e}$ $g$ representa o gasto público. 
Porque o que caracteriza um governo "durão" $(T)$ é um menor peso atribuído ao gasto público comparativamente a um governo "fraco" $(W)$, temos que $\theta_{T}$ $<\theta_{W}$.

\subsection{Tecnologia e restrição orçamentária}

As receitas do governo são dadas por:

$$
\tau=\bar{\tau}+\gamma\left(m_{t}-m_{t}^{E}\right)
$$

Onde a constante, $\bar{\tau}$, representa o nível "normal" de arrecadação; $\left(m_{t}-m_{t}^{E}\right)$ é a surpresa inflacionária, e $\gamma$ é um parâmetro de elasticidade que visa capturar o efeito combinado da melhora na atividade econômica de curto prazo que resulta de uma surpresa inflacionária com o quanto desta melhora se transforma em maior arrecadação de impostos.

A hipótese de que a surpresa inflacionária afeta positivamente a atividade econômica - que é a base da formulação da Curva de Phillips aumentada por expectativas - em geral é justificada com base nos modelos de salários nominais rígidos a la Fisher (1977) ou Taylor (1979). De acordo com estes, aumentos não esperados dos preços levam a reduções do salário real, maior demanda por trabalho e, conseqüentemente, a uma maior produção final de bens. Note-se, no entanto, que aumentos não esperados dos preços podem também levar a um acréscimo não esperado de outros custos de produção (como matérias-primas, por exemplo) e, portanto, para que surpresas inflacionárias estimulem a atividade no curto prazo, é preciso que o trabalho seja um insumo de produção significativo na estrutura de custos da firma. ${ }^{7}$ Felizmente, esta dubiedade é resolvida na arena empírica, já que é possível encontrar nos dados uma relação sistemática entre surpresa inflacionária e ritmo de atividade no curto prazo. ${ }^{8}$

Em $t=1$, temos que a restrição orçamentária do governo ${ }^{9}$ é (como hipótese simplificadora, assume-se que a dívida pública vence apenas no segundo período, e que o governo não pode se endividar no primeiro período):

$$
g_{1}=\bar{\tau}+\gamma\left(m_{1}-m_{1}^{E}\right)
$$

\footnotetext{
${ }^{7}$ Outro possível canal é via diminuição do fardo real de uma dívida corporativa prefixada. Quando a dívida é prefixada e a inflação sobe além do esperado, a restrição orçamentária da firma é relaxada. Em um mundo onde os mercados de capitais são imperfeitos, esta "folga" financeira pode significar maiores investimentos produtivos.

${ }^{8}$ Ver Barro $(1977,1978)$ e Alesina et alii (1997) no contexto de ciclos monetários partidários.

${ }^{9}$ Onde $g$ representa o gasto real em cada período.
} 
Em $t=2$, com o vencimento da dívida herdada, $b$, a restrição orçamentária passa a ser:

$$
g_{2}=\bar{\tau}+\gamma\left(m_{t}-m_{t}^{E}\right)-\left(1+r\left(m_{1}\right)\right) b
$$

Postulamos uma forma reduzida para a taxa real de juros, $1+r\left(m_{1}\right)$, decompondo-a em um componente fixo de longo prazo, $R$, e um componente monetário de curto prazo. Vale dizer que em um modelo neoclássico padrão com preços flexíveis, o juro real é dado apenas pela taxa de impaciência do agente representativo. Portanto, para justificar a hipótese de que o juro real responde a surpresas inflacionárias no curto prazo é preciso mais uma vez deixar explícito que assumimos no presente contexto que a economia está sujeita a algum grau de rigidez nominal. A evidência empírica sinaliza que esta é de fato uma boa hipótese (mais uma vez, de curto prazo), dado que "choques" monetários (o equivalente a surpresa inflacionária no modelo aqui apresentado) afetam tanto a taxa de juro real como a atividade econômica (ver, por exemplo, Christiano et alii (1998)). ${ }^{10}$

$$
1+r\left(m_{1}\right)=R-\xi \cdot\left(m_{1}-m_{1}^{E}\right)
$$

onde $\xi$ é a elasticidade do juro real a surpresas inflacionárias.

\subsection{Benchmark: equilíbrio com informação perfeita}

Quando não há assimetrias de informação, ambos tipos de governo, tomando as expectativas como dadas escolhem $m_{1}$ e $m_{2}$ de modo a maximizar:

$$
\begin{aligned}
U_{i}= & \theta_{i}\left(\bar{\tau}+\gamma\left(m_{1}-m_{1}^{E}\right)\right)-\frac{m_{1}^{2}}{2}+ \\
& \beta\left\{\theta_{i}\left(\bar{\tau}+\gamma\left(m_{2}-m_{2}^{E}\right)-\left(R-\xi\left(m_{1}-m_{1}^{E}\right)\right) b\right)-\frac{m_{2}^{2}}{2}\right\}
\end{aligned}
$$

As condições de primeira ordem deste problema são:

Para o tipo-T:

$$
M_{1}^{T}=(\gamma+\beta b \xi) \theta_{T}
$$

\footnotetext{
${ }^{10}$ Uma outra possível explicação se baseia no fato de parte da dívida pública ser prefixada. Os juros implícitos nestes títulos são fixados ex-ante com base nas expectativas de inflação dos agentes. Se estas expectativas são frustradas via surpresa inflacionária, o juro real ex-post pago por estes papéis é reduzido, e a restrição orçamentária do governo aliviada.
} 


$$
M_{2}^{T}=\gamma \theta_{T}
$$

Para o tipo-W:

$$
\begin{gathered}
M_{1}^{W}=(\gamma+\beta b \xi) \theta_{W} \\
M_{2}^{W}=\gamma \theta_{W}
\end{gathered}
$$

Denominamos $M_{t}^{T}$ e $M_{t}^{W}$ como as políticas preferidas pelos respectivos tipos. Como esperado, a inflação cresce com a dívida pública, com a elasticidade do juro real em relação à inflação, e com a importância atribuída aos gastos públicos. Note-se também que $\theta_{T}<\theta_{W}$ implica em $M_{1 ; 2}^{T}<M_{1 ; 2}^{W}$.

Aqui, é importante enfatizar que, em equilíbrio, as ações do governo são plenamente antecipadas e, portanto, o fato de o governo não conseguir impor uma inflação nula (devido ao clássico problema de inconsistência temporal) gera apenas perdas de bem-estar. ${ }^{11}$

Por fim, é interessante notar, observando (6) e (8), que na ausência de assimetrias informacionais, há uma relação continuamente positiva entre a inflação do primeiro período e o tamanho da dívida pública.

\subsection{Informação imperfeita e sinalização}

Nesta seção, derivamos as principais proposições do artigo. Como as preferências do governo são agora informação privada, as expectativas de inflação dos agentes são formadas com base na probabilidade prior de o governo ser do tipo durão, $p$, e também com base na inflação observada para o primeiro período. ${ }^{12}$ Para garantir consistência temporal, o jogo é resolvido empregando indução retroativa.

Mais uma vez, como no segundo período não há espaço para manobras estratégicas, ambos os tipos escolhem sempre a inflação $M_{2}^{i}=\gamma \cdot \theta_{i}$. A seguir, investigamos a possibilidade de equilíbrios agregador e separador no primeiro período.

\footnotetext{
${ }^{11} \mathrm{Em}$ modelos onde a economia é atingida por choques não antecipados pelo setor privado, este resultado já não é mais inequívoco. Ver, por exemplo, Alesina e Grilli (1992).

${ }^{12}$ Note-se que o governo entrante é de fato do tipo conservador. Seu problema principal é conseguir convencer o público, que não observa diretamente suas preferências, de suas credenciais antiinflacionárias.
} 


\section{Equilíbrio separador}

Em um equilíbrio separador, o tipo-T é capaz de se diferenciar, perante o público, de um governo do tipo-W. Segue-se que $p_{2}=1$ e $m_{2}^{E}=\gamma \theta_{T}$. Notese também que, neste equilíbrio plenamente revelador, como o tipo-W é incapaz de esconder suas credenciais, ele não colhe qualquer benefício em desviar de sua política preferida, $M_{1}^{W}$.

Assim, denominamos o par $\left\{m_{s} ; M_{1}^{W}\right\}$ como nosso candidato a equilíbrio separador. Para que este seja de fato um equilíbrio, é preciso que o tipo-T não tenha incentivos em desviar unilateralmente para $M_{1}^{T}$ (desperdiçando assim sua reputação, $\left.p_{2}=0\right)$ e também que o tipo-W esteja melhor implementando sua política preferida (que implica $p_{2}=0$ ) do que imitando o tipo-T com uma inflação $m_{s}$ (que levaria a $p_{2}=1$ ).

Assim, $\left\{m_{s} ; M_{1}^{W}\right\}$ será um equilíbrio separador se: ${ }^{13}$

$$
U_{T}^{s}>U_{T}^{d} \text { e } U_{W}^{d}>U_{W}^{s}
$$

em que:

$$
\begin{aligned}
U_{T}^{s}= & \theta_{T}\left(\bar{\tau}+\gamma\left(m_{s}-m_{1}^{E}\right)\right)-\frac{m_{s}^{2}}{2}+ \\
& \beta\left\{\theta_{T}\left(\bar{\tau}+\gamma\left(\gamma \theta_{T}-\gamma \theta_{T}\right)-\left(R-\xi\left(m_{s}-m_{1}^{E}\right)\right) b\right)-\frac{\left(\gamma \theta_{T}\right)^{2}}{2}\right\} \\
U_{T}^{d}= & \theta_{T}\left(\bar{\tau}+\gamma\left(M_{1}^{T}-m_{1}^{E}\right)\right)-\frac{\left(M_{1}^{T}\right)^{2}}{2}+ \\
& \beta\left\{\theta_{T}\left(\bar{\tau}+\gamma\left(\gamma \theta_{T}-\gamma \theta_{W}\right)-\left(R-\xi\left(M_{1}^{T}-m_{1}^{E}\right)\right) b\right)-\frac{\left(\gamma \theta_{T}\right)^{2}}{2}\right\} \\
U_{W}^{d}= & \theta_{W}\left(\bar{\tau}+\gamma\left(M_{1}^{W}-m_{1}^{E}\right)\right)-\frac{\left(M_{1}^{W}\right)^{2}}{2}+ \\
& \beta\left\{\theta_{W}\left(\bar{\tau}+\gamma \cdot\left(\gamma \cdot \theta_{W}-\gamma \theta_{W}\right)-\left(R-\xi\left(M_{1}^{W}-m_{1}^{E}\right)\right) b\right)-\frac{\left(\gamma \theta_{W}\right)^{2}}{2}\right\}
\end{aligned}
$$

\footnotetext{
${ }^{13}$ Os sobrescritos $d, p$ e $s$ denotam respectivamente "desvio"; equilíbrio agregador e equilíbrio separador.
} 


$$
\begin{aligned}
U_{W}^{s}= & \theta_{W}\left(\bar{\tau}+\gamma\left(m_{s}-m_{1}^{E}\right)\right)-\frac{\left(m_{s}\right)^{2}}{2}+ \\
& \beta\left\{\theta_{W}\left(\bar{\tau}+\gamma\left(\gamma \theta_{W}-\gamma \theta_{T}\right)-\left(R-\xi\left(m_{s}-m_{1}^{E}\right)\right) b\right)-\frac{\left(\gamma \theta_{W}\right)^{2}}{2}\right\}
\end{aligned}
$$

Para o tipo- $\mathrm{T}$ :

$$
\begin{gathered}
U_{T}^{s}-U_{T}^{d}=\theta_{T} \gamma\left(m_{s}-M_{1}^{T}\right)+\frac{\left(M_{1}^{T}\right)^{2}}{2}-\frac{m_{s}^{2}}{2}+\beta \gamma^{2} \theta_{T} \Delta \theta+\beta \theta_{T} b \xi\left(m_{s}-M_{1}^{T}\right) \\
U_{T}^{s}-U_{T}^{d}=\left(m_{s}-M_{1}^{T}\right) \underbrace{\left(\theta_{T} \gamma+\beta \theta_{T} b \xi\right)}_{M_{1}^{T}}+\frac{\left(M_{1}^{T}\right)^{2}}{2}-\frac{m_{s}^{2}}{2}+\beta \gamma^{2} \theta_{T} \Delta \theta \\
U_{T}^{s}>U_{T}^{d} \Leftrightarrow m_{s}^{2}-2 M_{1}^{T} m_{s}+\left(M_{1}^{T}\right)^{2}-2 \beta \gamma^{2} \theta_{T} \Delta \theta<0
\end{gathered}
$$

Implicando em:

$$
m_{s}>M_{1}^{T}-\gamma \sqrt{2 \beta \Delta \theta \theta_{T}}
$$

Para o tipo-W:

$$
\begin{gathered}
U_{W}^{d}-U_{W}^{s}=\theta_{W} \gamma\left(M_{1}^{W}-m_{s}\right)+\frac{m_{s}^{2}}{2}-\frac{\left(M_{1}^{W}\right)^{2}}{2}-\beta \gamma^{2} \theta_{W} \Delta \theta+\beta \theta_{W} b \xi\left(M_{1}^{W}-m_{s}\right) \\
U_{W}^{d}-U_{W}^{s}=\left(M_{1}^{W}-m_{s}\right)(\underbrace{\left.\theta_{W} \gamma+\beta \theta_{W} b \xi\right)}_{M_{1}^{W}}+\frac{m_{s}^{2}}{2}-\frac{\left(M_{1}^{W}\right)^{2}}{2}-\beta \gamma^{2} \theta_{W} \Delta \theta \\
U_{W}^{d}>U_{W}^{s} \Leftrightarrow m_{s}^{2}-2 M_{1}^{W} m_{s}+\left(M_{1}^{W}\right)^{2}-2 \beta \gamma^{2} \theta_{W} \Delta \theta>0 \text { Resultando em: } \\
m_{s}<M_{1}^{W}-\gamma \sqrt{2 \beta \Delta \theta \theta_{W}}
\end{gathered}
$$

Portanto, um equilíbrio separador será factível se:

$$
M_{1}^{W}-\gamma \sqrt{2 \beta \Delta \theta \theta_{W}}>M_{1}^{T}-\gamma \sqrt{2 \beta \Delta \theta \theta_{T}}
$$

or

$$
\gamma \sqrt{2 \beta \Delta \theta}\left(\sqrt{\theta_{W}}-\sqrt{\theta_{T}}\right)<(\gamma+\beta b \xi) \Delta \theta
$$




$$
b>\frac{\gamma}{\beta \xi}\left[\frac{\sqrt{2 \beta}\left(\sqrt{\theta_{W}}-\sqrt{\theta_{T}}\right)}{\sqrt{\Delta \theta}}-1\right]=b_{l}^{t h r}
$$

A intuição aqui é direta: se a dívida herdada não é alta o suficiente (maior que $b_{l}^{\text {thr }}$ ), um governo "fraco" tem uma perda apenas pequena - em termos de menor gasto público no segundo período - em imitar um governo "durão", e opta, portanto, em colher os benefícios de uma inflação esperada mais baixa. Por esta razão, é impossível para o "durão" sinalizar suas credenciais antiinflacionárias quando a dívida é pequena.

Já quando a dívida é superior a $b_{l}^{t h r}$, o tipo-T poderá (e preferirá se $b<b_{h}^{t h r}$, deduzido abaixo) implementar $m_{s}^{*}=M_{1}^{W}-\gamma \sqrt{2 \beta \Delta \theta \theta_{W}}\left(<M_{1}^{T}\right),{ }^{14}$ separando-se, sob os olhos do público, de um governo tipo-W. Neste caso, a inflação esperada será $m_{2}^{E}=\gamma \theta_{T}$ e a inflação do primeiro período será de $m_{s}^{*}$ (menor, portanto, que a prevalescente no caso de informação perfeita).

\section{Um equilíbrio de agregador}

Nesta subseção, checamos a possibilidade de um equilíbrio agregador em $M_{1}^{T}$. Vale dizer que é possível que existam outros equilíbrios desta natureza para valores de $m_{1} \neq M_{1}^{T}$. No entanto, neste artigo nos limitaremos a investigar a possibilidade de agregação (pooling) apenas em $M_{1}^{T} \cdot{ }^{15}$

Em um equilíbrio agregador, $m_{2}^{E}=\gamma\left[p \theta_{T}+(1-p) \theta_{W}\right]$. Mais uma vez, caso desvios deste equilíbrio gerem perda total de reputação, um tipo-W perseguirá sua política preferida caso opte por desviar. Isto posto, um governo tipo-W mimetizará um "durão" (em $\left.M_{1}^{T}\right)$ apenas se $U_{W}^{p}>U_{W}^{d}$.

onde:

$$
\begin{aligned}
U_{W}^{d}= & \theta_{W}\left(\bar{\tau}+\gamma\left(M_{1}^{W}-m_{1}^{E}\right)\right)-\frac{\left(M_{1}^{W}\right)^{2}}{2}+ \\
& \beta\left\{\theta_{W}\left(\bar{\tau}+\gamma\left(\gamma \theta_{W}-\gamma \theta_{W}\right)-\left(R-\xi\left(M_{1}^{W}-m_{1}^{E}\right)\right) b\right)-\frac{\left(\gamma \theta_{W}\right)^{2}}{2}\right\}
\end{aligned}
$$

\footnotetext{
${ }^{14} E$ É fácil mostrar que esta última desigualdade vale para todo o intervalo onde um equilíbrio separador é factível.

${ }^{15}$ Contudo, neste modelo, soa ao menos natural investigar a possibilidade de pooling na inflação preferida do governo durão.
} 


$$
\begin{aligned}
U_{W}^{p}= & \theta_{W}\left(\bar{\tau}+\gamma\left(M_{1}^{T}-m_{1}^{E}\right)\right)-\frac{\left(M_{1}^{T}\right)^{2}}{2}+ \\
& \beta\left\{\theta _ { W } \left(\bar{\tau}+\gamma\left(\gamma \theta_{W}-\gamma\left(p \theta_{T}+(1-p) \theta_{W}\right)\right)\right.\right. \\
& \left.\left.-\left(R-\xi\left(M_{1}^{T}-m_{1}^{E}\right)\right) b\right)-\frac{\left(\gamma \theta_{W}\right)^{2}}{2}\right\}
\end{aligned}
$$

Após alguma manipulação algébrica, chega-se a:

$$
U_{W}^{P}>U_{W}^{d} \Rightarrow p>\frac{\Delta \theta(\gamma+\beta b \xi)^{2}}{2 \beta \gamma^{2} \theta_{W}}
$$

A intuição aqui é a seguinte: como $p_{2}=p$ - dado que os agentes em um equilíbrio agregador não extraem nenhuma informação adicional ao observar $M_{1}^{T}$ - somente será interessante para o tipo-W imitar o tipo-T quando a probabilidade prior de o governo ser "durão" for suficientemente alta.

Note-se também que, como $p$ é uma medida de probabilidade, a desigualdade acima implica necessariamente que $\frac{\Delta \theta(\gamma+\beta b \xi)^{2}}{2 \beta \gamma^{2} \theta_{W}}<1$. Após algumas manipulações álgebricas, chega-se facilmente a:

$$
b<\frac{\gamma}{\beta \xi}\left(\frac{\sqrt{2 \beta} \sqrt{\theta_{W}}}{\sqrt{\Delta \theta}}-1\right)=b_{h}^{t h r}
$$

Portanto, um equilíbrio agregador somente será possível quando a dívida for suficientemente baixa. Neste tipo de equilíbrio, um tipo-T escolhe uma inflação $M_{1}^{T}$ (dado que não há ganhos em ser ainda mais durão $\left(m_{s}\right)$ neste caso) no primeiro período, e as expectativas de inflação passam a ser $m_{2}^{E}=\gamma\left[p \theta_{T}+(1-p) \theta_{W}\right]$. Assim, tanto a inflação realizada como a esperada são mais elevadas que em um equilíbrio separador.

Finalmente, dado que $b_{h}^{t h r}>b_{l}^{t h r}$, existe uma região não vazia onde (se a prior é suficientemente alta) ambos equilíbrios - pooling e separador - são eventos possíveis.

Proposição 1: Um equilíbrio agregador em $M_{1}^{T}$ é "não-intuitivo"16 para $b_{l}^{\text {thr }}<$ $b<b_{h}^{t h r}$. Isto porque $\exists m_{1}$ tal que $U_{T}\left(m_{1} \mid p_{2}=1\right)>U_{T}\left(m_{1}=M_{1}^{T} \mid p_{2}=p\right) e$ $U_{W}\left(m_{1} \mid p_{2}=1\right)<U_{W}\left(m_{1}=M_{1}^{T} \mid p_{2}=p\right) .{ }^{17}$

\footnotetext{
${ }^{16} \mathrm{O}$ conceito de refinemento aqui empregado é o critério intuitivo de Cho e Kreps (1987).

${ }^{17}$ A lógica da prova é muito similar a empregada em Rogoff (1990). Em particular, ver a nota de rodapé 16 daquele artigo.
} 
Prova: Ver apêndice.

\section{Discussão}

Nesta seção, resumimos e discutimos os resultados até aqui derivados. Com base no acima exposto, sabemos que:

(i) Se $b<b_{l}^{t h r}$, não haverá equilíbrio separador e ambos tipos implementarão $M_{1}^{T}$ em um equilíbrio agregador. Assim, a inflação esperada será de $m_{2}^{E}=$ $\gamma\left[p \theta_{T}+(1-p) \theta_{W}\right]$, e realizada no primeiro período $M_{1}^{T}$.

(ii) Se $b_{l}^{t h r}<b<b_{h}^{t h r}$, apesar de um equilíbrio agregador ser possível, ele (ao contrário do separador) é não-intuitivo. Portanto, para este intervalo $m_{1}=$ $m_{s}^{*}$ e $m_{2}^{E}=\gamma \theta_{T}$ (menor que no caso (i).

(iii) Quando $b_{h}^{t h r}<b$, o efeito de dominância fiscal prevalece (dívida mais alta leva inequivocamente a mais inflação). Nesta região, o endividamento é tão elevado que ambos os tipos preferem implementar sua política preferida. Portanto, se o governante é do tipo-T, $m_{1}=M_{1}^{T}$ e $m_{2}^{E}=\gamma \theta_{T}$.

Com base nestas considerações, enunciamos a proposição abaixo.

Proposição 2: Quando a dívida herdada é inferior a $b_{l}^{\text {thr }}$, as expectativas de inflação são mais altas do que quando ela é superior a este valor. Mais ainda, quando $b_{l}^{\text {thr }}<b<b_{h}^{\text {thr }}$, a inflação realizada é inferior a do caso com informação perfeita, o que significa que niveis intermediários de endividamento ajudam a diminuir o viés inflacionário. Finalmente, se $b_{h}^{\text {thr }}<b$, o efeito de dominância fiscal prevalesce sobre o de sinalização. 
A figura abaixo, ao colocar inflação esperada em um eixo, e o nível da dívida em outro, ilustra que, quando atribuímos um papel importante para o efeito de sinalização (o que parece crucial no caso brasileiro), não é mais verdade que a expectativa de inflação será maior para patamares mais elevados de endividamento.

Figura 1

Expectativa de inflação e dívida

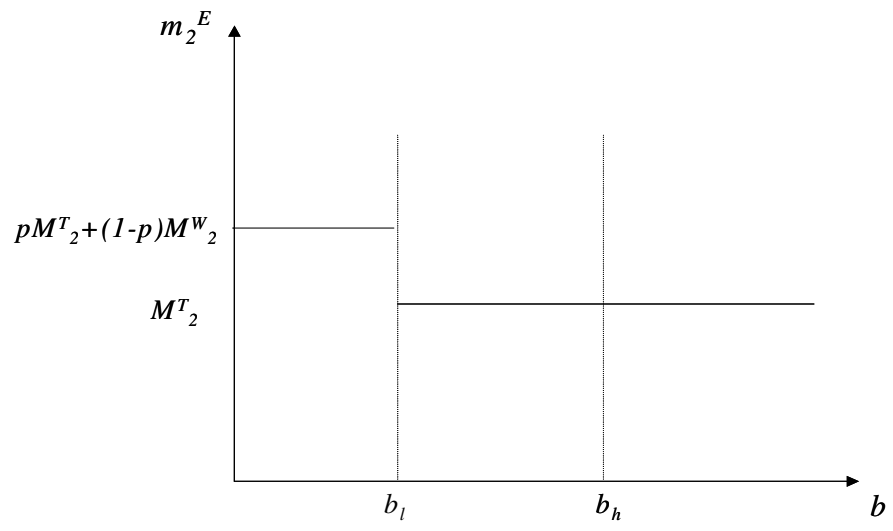

Em relação à inflação do primeiro período, vimos que ela será dada por $M_{1}^{T}$ se $b<b_{l}^{t h r} ; m_{s}^{*}$ se $b_{l}^{t h r}<b<b_{h}^{t h r}$ e, mais uma vez, $M_{1}^{T}$ se $b_{h}^{t h r}<b$. Como se pode ver na figura abaixo, isto implica que a correlação positiva entre dívida e inflação deixa de ser contínua. Em particular, note-se que para a região $\left(b_{l}^{t h r} ; b_{A}\right]$, apesar de a inflação ser crescente na dívida, ela é menor que a inflação em $b_{l}^{\text {thr }}$, qual seja, $M_{1}^{T}\left(b_{l}^{t h r}\right) .{ }^{18}$

\footnotetext{
${ }^{18}$ A figura é desenhada assumindo $\gamma=1$, caso no qual é fácil mostrar que vale a igualdade $M^{T}\left(b_{h}^{t h r}\right)=m^{s}\left(b_{h}^{t h r}\right)$. Quando $\gamma<1$, a única mudança é que $M^{T}\left(b_{h}^{t h r}\right)>m^{s}\left(b_{h}^{t h r}\right)$.
} 
Figura 2

Inflação realizada e dívida

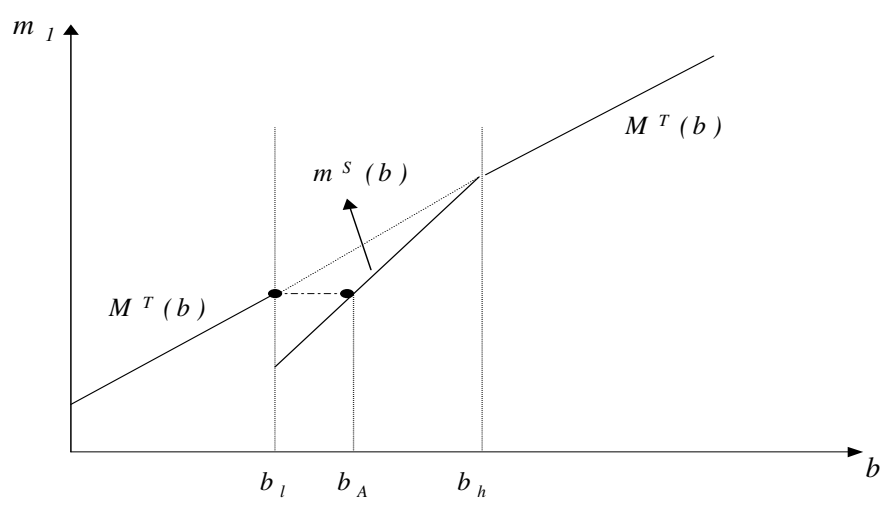

Proposição 3: A relação positiva entre dívida e inflação deixa de ser contínua quando há assimetria de informação em relação às preferências do governo.

\section{Conclusão}

Neste artigo, desenvolvemos um modelo onde coexistem efeitos de dominância fiscal (dívida mais alta pressionando a inflação para cima) e de sinalização (quando a dívida cresce, o governo vê a possibilidade de se separar jogando a inflação para baixo). Contrariamente ao que argumentam os defensores da tese de dominância fiscal, quando existe assimetria de informação, as correlações entre o tamanho da dívida pública e as inflações realizada e esperada, deixam de ser trivialmente positivas.

Demonstramos que, como um governo "durão" só consegue sinalizar suas credenciais quando a dívida herdada está acima de um certo patamar (endogenamente determinado), a relação da inflação e da inflação esperada com o tamanho do endividamento pode se tornar negativa.

Em resumo, atribuindo papel fundamental para o problema de assimetria de informação prevalescente na transição do goveno FHC para o governo Lula, este artigo provê uma justificativa para o fato de as expectativas de inflação terem declinaram vigorosamente em 2003, a despeito dos efeitos negativos relacionados à aritmética desagradável derivados de um endividamento elevado. 


\section{Referências}

Alesina, A. \& Grilli, V. (1992). The European Central Bank: Reshaping monetary policy in Europe. In Canzoneri, M., Grilli, V., \& Masson, P., editors, Establishing a Central Bank: Issues in Europe and Lessons from US. Cambridge University Press.

Alesina, A., Roubini, N., \& Cohen, G. (1997). Political Cycles and the Macroeconomy. The MIT Press.

Backus, D. \& Driffil, J. (1985). Inflation and reputation. American Economic Review, 74:530-538.

Barro, R. (1977). Unanticipated money growth and unemployment in the United States. American Economic Review, 67:101-115.

Barro, R. (1978). Unanticipated money, output and the price level in the United States. Journal of Political Economy, 86:549-580.

Benigno, P. \& Missale, A. (2004). High public debt in currency crises: Fundamentals versus signalling effects. Journal of International Money and Finance, $23(2)$.

Blanchard, O. (2004). Fiscal dominance and inflation targeting: Lessons from Brazil. NBER Working Paper 10389.

Cho, I. \& Kreps, D. (1987). Signalling games and stable equilibria. Quarterly Journal of Economics, 102:179-221.

Christiano, L., Eichenbaum, M., \& Evans, C. (1998). Monetary policy shocks: What have we learned, and to what end? NBER Working Paper 6400.

Drazen, A. \& Masson, P. (1994). Credibility of policies versus credibility of policymakers. Quarterly Journal of Economics, 109.

Fisher, S. (1977). Long-term contracts, rational expectations, and the optimal money supply rule. Journal of Political Economy, 85:191-205.

Rogoff, K. (1985). The optimal degree of commitment to an intermediate monetary target. The Quarterly Journal of Economics, 100:1169-90.

Rogoff, K. (1990). Political budget cycles. American Economic Review, 80:21-36. 
Sargent, T. \& Wallace, N. (1981). Some unpleasant monetaristic arithmetics. Federal Reserve Bank of Minneapolis Quarterly Review.

Taylor, J. (1979). Staggered wage setting in a macro model. American Economic Review, 69:108-113.

Vickers, J. (1986). Signalling in a model of monetary policy with incomplete information. Oxford Economic Papers, 38:443-455. 


\section{Apêndice}

Aqui apresentamos a prova da proposição 1.

Prova: Tome, por exemplo, $m_{1}=m_{s}$.

Parte 1: Primeira Desigualdade

$$
\begin{aligned}
& U_{T}^{s}=\theta_{T}\left(\bar{\tau}+\gamma\left(m_{s}-m_{1}^{E}\right)\right)-\frac{m_{s}^{2}}{2}+ \\
& \beta\left\{\theta_{T}\left(\bar{\tau}+\gamma\left(\gamma \theta_{T}-\gamma \theta_{T}\right)-\left(R-\xi\left(m_{s}-m_{1}^{E}\right)\right) b\right)-\frac{\left(\gamma \theta_{T}\right)^{2}}{2}\right\} \\
& U_{T}^{p}=\theta_{T}\left(\bar{\tau}+\gamma\left(M_{1}^{T}-m_{1}^{E}\right)\right)-\frac{\left(M_{1}^{T}\right)^{2}}{2}+ \\
& \beta\left\{\theta_{T}\left(\bar{\tau}+\gamma\left(\gamma \theta_{T}-\gamma p \theta_{T}-\gamma(1-p) \theta_{W}\right)-\left(R-\xi\left(M_{1}^{T}-m_{1}^{E}\right)\right) b\right)-\frac{\left(\gamma \theta_{T}\right)^{2}}{2}\right\} \\
& U_{T}^{s}-U_{T}^{p}>0 \Leftrightarrow\left(m_{s}-M_{1}^{T}\right) M_{1}^{T}+\beta \gamma^{2}(1-p) \Delta \theta \theta_{T}+\frac{\left(M_{1}^{T}\right)^{2}}{2}-\frac{m_{s}^{2}}{2}>0 \Leftrightarrow \\
& \left(m_{s}-M_{1}^{T}\right)\left(M_{1}^{T}-\left(\frac{M_{1}^{T}+m_{s}}{2}\right)\right)+\beta \gamma^{2}(1-p) \Delta \theta \theta_{T}>0 \Leftrightarrow \\
& -\frac{\left(m_{s}-M_{1}^{T}\right)^{2}}{2}+\beta \gamma^{2} \Delta \theta \theta_{T}>\beta \gamma^{2} \Delta \theta \theta_{T} p \Leftrightarrow \\
& p<1-\frac{\left(m_{s}-M_{1}^{T}\right)^{2}}{2 \beta \gamma^{2} \cdot \Delta \theta \theta_{T}} \Rightarrow 1-\frac{\left(m_{s}-M_{1}^{T}\right)^{2}}{2 \beta \gamma^{2} \Delta \theta \theta_{T}}>0 \Leftrightarrow 2 \beta \gamma^{2} \Delta \theta \theta_{T}>\left(m_{s}-M_{1}^{T}\right)^{2}
\end{aligned}
$$

Lembrando que $m_{s}=M_{1}^{W}-\gamma \sqrt{2 \beta \Delta \theta \theta_{W}}$, temos:

$$
\left(m_{s}-M_{1}^{T}\right)^{2}=\left(\Delta \theta(\gamma+\beta b \xi)-\gamma \sqrt{2 \beta \Delta \theta \theta_{W}}\right)^{2}
$$

Assim:

$2 \beta \gamma^{2} \Delta \theta \theta_{T}>\Delta \theta^{2}(\gamma+\beta b \xi)^{2}-2 \gamma \Delta \theta(\gamma+\beta b \xi) \sqrt{2 \beta \Delta \theta \theta_{W}}+\gamma^{2} 2 \beta \Delta \theta \theta_{W}$

Definindo: $\Psi \approx(\gamma+\beta b \xi)$ e após alguma álgebra:

$\Psi^{2} \Delta \theta-2 \gamma \Psi \sqrt{2 \beta \Delta \theta \theta_{W}}+2 \beta \gamma^{2} \Delta \theta<0$ 
Tal desigualdade será satisfeita quando:

$$
\frac{\gamma}{\beta \xi}\left[\frac{\sqrt{2 \beta}\left(\sqrt{\theta_{W}}-\sqrt{\theta_{T}}\right)}{\sqrt{\Delta \theta}}\right]-1\left(=b_{l}^{\text {thr }}\right)<b<\frac{\gamma}{\beta \xi}\left[\frac{\sqrt{2 \beta}\left(\sqrt{\theta_{W}}+\sqrt{\theta_{T}}\right)}{\sqrt{\Delta \theta}}-1\right]\left(>b_{h}^{\text {thr }}\right)
$$

\section{Parte 2:}

$$
\begin{aligned}
& U_{W}^{p}=\theta_{W}\left(\bar{\tau}+\gamma\left(M_{1}^{T}-m_{1}^{E}\right)\right)-\frac{\left(M_{1}^{T}\right)^{2}}{2}+ \\
& \beta\left\{\theta _ { W } \left(\bar{\tau}+\gamma\left(\gamma \theta_{W}-\gamma\left(p \theta_{T}+(1-p) \theta_{W}\right)\right)-\right.\right. \\
& \left.\left.\left(R-\xi\left(M_{1}^{T}-m_{1}^{E}\right)\right) b\right)-\frac{\left(\gamma \theta_{W}\right)^{2}}{2}\right\} \\
& U_{W}^{s}=\theta_{W}\left(\bar{\tau}+\gamma\left(m_{s}-m_{1}^{E}\right)\right)-\frac{\left(m_{s}\right)^{2}}{2}+ \\
& \beta\left\{\theta_{W}\left(\bar{\tau}+\gamma\left(\gamma \theta_{W}-\gamma \theta_{T}\right)-\left(R-\xi\left(m_{s}-m_{1}^{E}\right)\right) b\right)-\frac{\left(\gamma \theta_{W}\right)^{2}}{2}\right\} \\
& U_{W}^{p}-U_{W}^{s}>0 \Leftrightarrow M_{1}^{W}\left(M_{1}^{T}-m_{s}\right)+\frac{\left(m_{s}\right)^{2}-\left(M_{1}^{T}\right)^{2}}{2}-\beta \gamma^{2} \Delta \theta \theta_{W} p>0
\end{aligned}
$$

Usando $m_{s}=M_{1}^{W}-\gamma \sqrt{2 \beta \Delta \theta \theta_{W}}$, chega-se, após manipulações entediantes:

$$
\begin{aligned}
& p<1-\frac{\Delta \theta(\gamma+\beta b \xi)^{2}}{2 \beta \gamma^{2} \theta_{W}} \Rightarrow 1-\frac{\Delta \theta(\gamma+\beta b \xi)^{2}}{2 \beta \gamma^{2} \theta_{W}}>0 \Leftrightarrow \\
& b<\frac{\gamma}{\beta \xi}\left[\frac{\sqrt{2 \beta} \sqrt{\theta_{W}}}{\sqrt{\Delta \theta}}-1\right]=b_{h}^{t h r}
\end{aligned}
$$

Assim, na região $\left(b_{l}^{\text {thr }} ; b_{h}^{\text {thr }}\right)$ um equilíbrio agregador é não-intuitivo. 